\title{
The static quark self-energy at large orders from NSPT
}

\section{Gunnar S. Bali, Clemens Bauer*}

Institut für Theoretische Physik, Universität Regensburg

D-93040 Regensburg, Germany

E-mail: gunnar.balidur.de

clemens.bauerephysik.uni-regensburg.de

\section{Antonio Pineda}

Grup de Física Teòrica, Universitat Autònoma de Barcelona,

E-08193 Bellaterra, Barcelona, Spain

E-mail: AntonioMique1.Pinedaduab.es

Using Numerical Stochastic Perturbation Theory (NSPT), we calculate the static self-energy of SU(3) gauge theory up to order $\alpha^{20}$. Simulations on a large set of different lattice volumes allow for a careful treatment of finite size effects. The resulting infinite volume perturbative series of the static self-energy is in remarkable agreement with the predicted asymptotic behaviour of high order expansions, namely with a factorial growth of perturbative coefficients known as renormalon.

XXIX International Symposium on Lattice Field Theory

July $10-162011$

Squaw Valley, Lake Tahoe, California

\footnotetext{
* Speaker.
} 


\section{Motivation}

Since the early days of quantum field theories, it has been argued that their perturbative weak coupling expansions are asymptotic [四]. Usually, the asymptotic behaviour is identified using semiclassical methods such as instantons. Asymptotically free theories with marginal operators constitute a special case. Examples of such theories are four-dimensional non-Abelian gauge theories or the two-dimensional $\mathrm{O}(N)$ model. For these the structure of the operator product expansion (OPE) is believed to give rise to a specific pattern of asymptotic divergence known as (infrared) renormalon [ [2, []] that cannot be obtained using standard semiclassical methods. In the two-dimensional $\mathrm{O}(N)$ model renormalon effects were found (though suppressed) in an explicit calculation in the large $N$ expansion [四]. For four-dimensional non-Abelian gauge theories no such proof exists. On the contrary, the possibility that the renormalon either does not exist or is very small has been raised in recent years, see e.g. [[G, 目].

At present, the only way to unambiguously settle this issue is by performing weak coupling expansions to sufficiently high orders in the strong coupling parameter $\alpha$. In particular, in SU(3) gauge theory, the problem has been addressed by simulations in lattice regularization, using $\mathrm{Nu}-$ merical Stochastic Perturbation Theory (NSPT) [ [-Q Q]. These simulations, that mainly focused on computing the plaquette and small Wilson loops to perturbative orders as high as $\alpha^{20}$ [एण]], so far had no success in uncovering a renormalon.

The situation radically changed recently when, for the first time, accurate agreement between the explicit computation of perturbative coefficients and the expectation from the renormalon analysis was reported [प]]. Here we discuss some aspects of this analysis where the comparison was performed using another observable, the static self-energy. This, on theoretical grounds, is far more suited for a renormalon study. The simulations were conducted on a large set of different lattice volumes, for orders as high as $\alpha^{20}$, and finite size effects were carefully investigated.

\section{Renormalon Theory}

In QCD, the expansion of a generic observable $K$ as a power series in the coupling $\alpha$,

$$
K=\sum_{n} k_{n} \alpha^{n}
$$

is believed not to be convergent but at best to be asymptotic. Technically, renormalons appear as singularities in the Borel plane, making a Borel summation impossible. The way in which the series $K$ diverges is closely tied to the OPE, commonly giving rise to terms $k_{n} \sim a_{d}^{n} n$ !, where $a_{d}$ is a constant. For small orders $n$, the successive contributions $k_{n} \alpha^{n}$ to the series reduce in size down to a minimum at $n_{0} \approx 1 /\left(\left|a_{d}\right| \alpha\right)$. The series should be truncated at $n_{0}$ and inevitably one has to deal with an ambiguity of the order of the minimum term, $k_{n_{0}} \alpha^{n_{0}} \sim \exp \left[-1 /\left(\left|a_{d}\right| \alpha\right)\right]$.

The OPE allows one to isolate the short (given by Wilson coefficients $C_{i}(q, \mu)$, where $i$ is the dimension) and long distance effects (matrix elements $\left\langle O_{i}(\mu, \Lambda)\right\rangle$ ) of an observable $R(q, \Lambda)$ by means of a factorization

$$
R=C_{0}(q, \mu)\left\langle O_{0}(\mu, \Lambda)\right\rangle+C_{d}(q, \mu)\left\langle O_{d}(\mu, \Lambda)\right\rangle\left(\frac{\Lambda}{q}\right)^{d}+\cdots
$$


Here, $\mu$ is the factorization scale separating the perturbative and low momentum scales $q$ and $\Lambda$ from each other: $q \gg \mu \gg \Lambda$.

For the plaquette, $\left\langle O_{0}\right\rangle=1$, followed by the dimension $d=4$ gluon condensate as the next higher dimensional non-vanishing operator. The lower bound for the accuracy of expanding $C_{0}$ is of $\mathscr{O}\left(\Lambda^{4} / q^{4}\right) \sim k_{n_{0}} \alpha^{n_{0}}$, the size of the minimum term, as can be seen from

$$
\left(\frac{\Lambda}{q}\right)^{d} \simeq \exp \left(-\frac{1}{\left|a_{d}\right| \alpha}\right), \quad \text { where } \quad\left|a_{d}\right|=\frac{\beta_{0}}{2 \pi d}, \quad \beta_{0}=11 .
$$

The (infrared) renormalon ambiguity of this perturbative series cancels that of the next order nonperturbative matrix element in eq. (2.2). Hence, the physical quantity $R$ is well-defined. It follows that series expansions with the smallest $d$ (and therefore $n_{0}$ ) are those in which one should be able to detect renormalons most easily (for $d=1$ four times "faster" than in the plaquette case). Among such candidates are the pole mass or the associated self-energy of a static source,

$$
\delta m=\frac{1}{a} \sum_{n \geq 0} c_{n} \alpha^{n+1}(1 / a) .
$$

$a^{-1}$, the inverse lattice spacing, serves as a UV-regulator. The large $n$ behaviour of the coefficients $c_{n}$ reads

$$
c_{n} \stackrel{n \rightarrow \infty}{=} N_{m}\left(\frac{\beta_{0}}{2 \pi}\right)^{n} \frac{\Gamma(n+1+b)}{\Gamma(1+b)}\left(1+\frac{b}{(n+b)} s_{1}+\cdots\right),
$$

where the coefficients $b$ and $s_{1}$ can be found in [12] and $N_{m}$ is a normalization constant that cancels when constructing ratios

$$
\frac{c_{n}}{c_{n-1}} \frac{1}{n}=\frac{\beta_{0}}{2 \pi}\left[1+\frac{b}{n}-\left(1-b s_{1}\right) \frac{b s_{1}}{n^{2}}+\mathscr{O}\left(\frac{1}{n^{3}}\right)\right] .
$$

\section{Numerical Simulation}

The simulations are performed within the framework of NSPT $[\square-Q]$ that allows for a direct determination of perturbative series coefficients. A key ingredient of NSPT is the Langevin update, for which we employ the $\mathscr{O}\left(\Delta \tau^{2}\right)$ integrator described in [[1]3, [4]]. Its convergence behaviour regarding the time step $\Delta \tau$ is remarkably flat, so that we can circumvent a $\Delta \tau$-extrapolation and work at a fixed value $\Delta \tau=0.05$. We implement periodic boundary conditions in time and twisted boundary conditions [5]-[18] in the three spatial directions, completely eliminating zero modes. Twists in two directions would have achieved this, too [10], but with reduced numerical stability.

Employing the Wilson gauge action, we calculate the temporal Polyakov line

$$
L^{(R)}\left(N_{S}, N_{T}\right)=\frac{1}{N_{S}^{3}} \sum_{\mathbf{n}} \frac{1}{d_{R}} \operatorname{tr}\left[\prod_{n_{4}=0}^{N_{T}-1} U_{4}^{R}(n)\right] .
$$

on hypercubic volumes with $N_{S}$ and $N_{T}$ lattice points in spatial and temporal directions, respectively. $R$ denotes the representation of the link $U_{\mu}^{R}(n)$, of dimension $d_{R}$, and we study both, the triplet and the octet cases. We repeat the measurements using stout-smeared [20] (smearing parameter $\rho=1 / 6$ ) links in the temporal direction. Altogether, this amounts to four distinct self-energies 


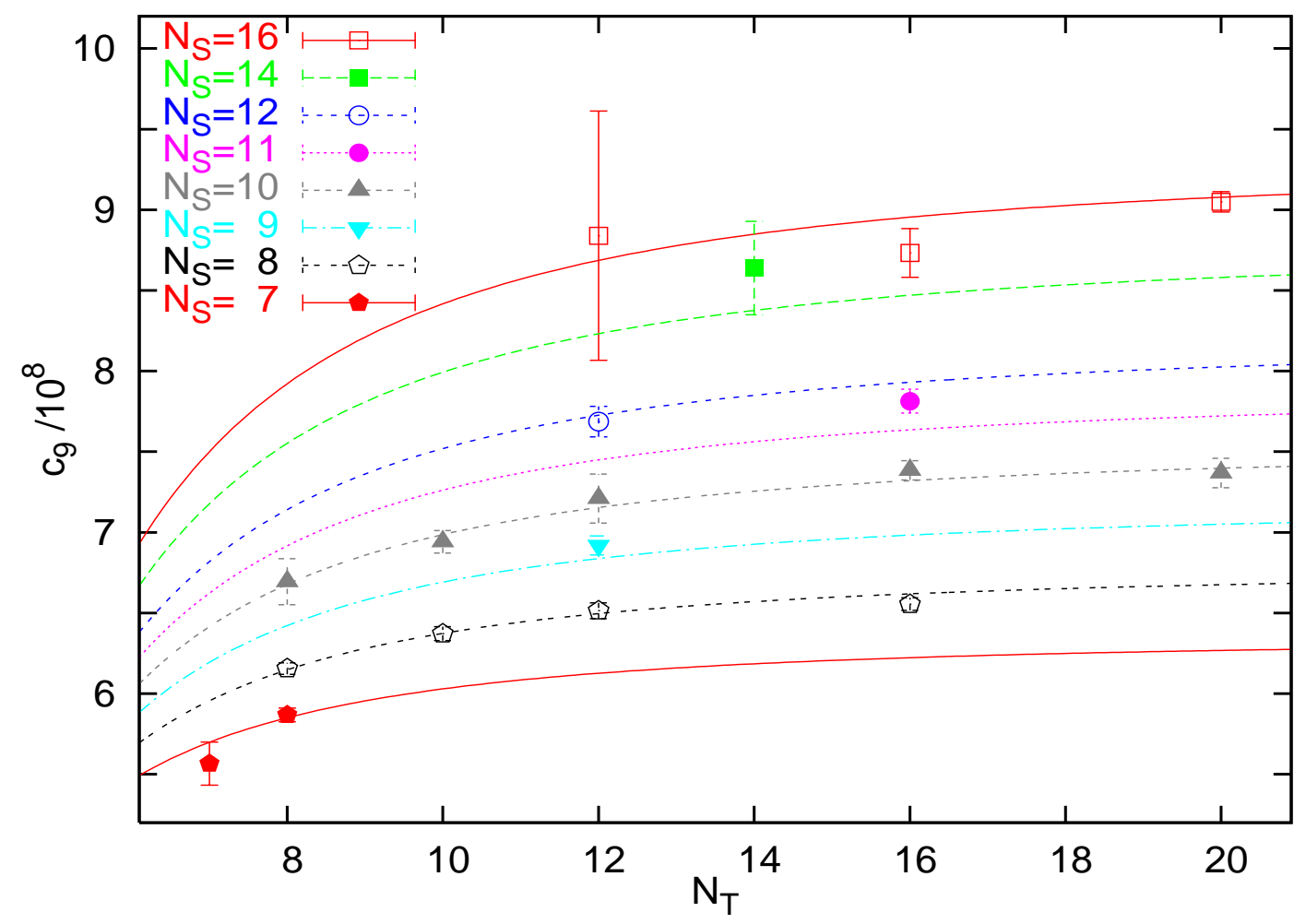

Figure 1: Comparison between the global fit and data for $n=9\left(\mathscr{O}\left(\alpha^{10}\right)\right)$.

(allowing for checks of Casimir scaling and universality) whose perturbative coefficients $c_{n}$ are linked to the Polyakov line via

$$
P\left(N_{S}, N_{T}\right)=-\frac{\ln \left\langle L\left(N_{S}, N_{T}\right)\right\rangle}{a N_{T}} \stackrel{N_{S}, N_{T} \rightarrow \infty}{\longrightarrow} \delta m .
$$

The dependence on $N_{T}$ and $N_{S}$ can be parameterized [Ш]] as

$$
\begin{aligned}
a P & =\sum_{n \geq 0}\left[c_{n} \alpha^{n+1}\left(a^{-1}\right)-\frac{f_{n}}{N_{S}} \alpha^{n+1}\left(\left(a N_{S}\right)^{-1}\right)+\cdots\right] \\
& =\sum_{n \geq 0}\left[c_{n}+\Delta_{n}^{(1)}\left(N_{S}\right)+\Delta_{n}^{(2)}\left(N_{S}, N_{T}\right)\right] \alpha^{n+1}\left(a^{-1}\right), \\
\Delta_{n}^{(1)} & =-\frac{1}{N_{S}}\left[f_{n}+\operatorname{logs}_{n}^{f}\left(N_{S}\right)\right], \\
\Delta_{n}^{(2)} & =\frac{1}{N_{T}^{2}}\left\{v_{n}-\frac{1}{N_{S}}\left[v_{n}+\delta v_{n}+\log s_{n}^{v}\left(N_{S}\right)\right]\right\}+\frac{1}{N_{S}^{2}}\left\{w_{n}-\frac{1}{N_{S}}\left[w_{n}+\delta w_{n}+\operatorname{logs} s_{n}^{w}\left(N_{S}\right)\right]\right\} .
\end{aligned}
$$

For sufficiently large $N_{T}$, the correction $\Delta_{n}^{(1)}$ dominates over $\Delta_{n}^{(2)}$, which comprises the leading $\mathscr{O}\left(1 / N_{T}^{2}, 1 / N_{S}^{2}\right)$ lattice artefacts. In contrast to the $1 / N_{T}^{2}$ correction terms, the $1 / N_{S}^{2}$ corrections do not have a significant effect on our fits. Therefore, we neglect the terms containing $w_{n}$ and $\delta w_{n}$.

The $\Delta_{n}^{(1)}$ contribution arises from interactions with mirror charges on lattice replica [R]], which result in an effective static potential between charges that are separated by distances $a N_{S}$, but without self-energies. Consequently, the high order behaviour of the coefficients $c_{n}$ and $f_{n}$ depends on 


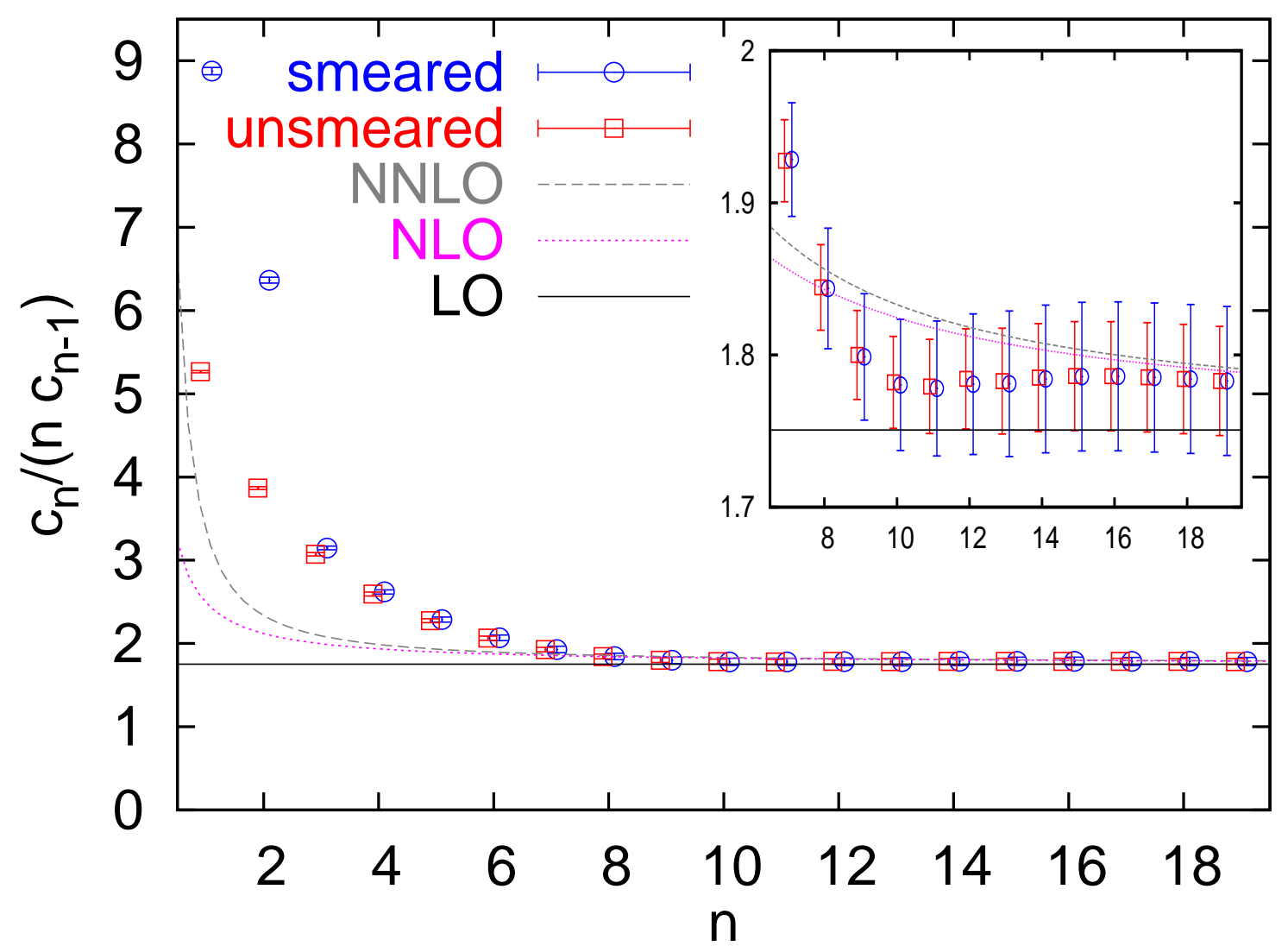

Figure 2: The ratios $c_{n} /\left(n c_{n-1}\right)$ for the smeared and unsmeared fundamental static self-energies, compared to the prediction eq. (2.6) at different orders of the $1 / n$ expansion.

the very same renormalon. Precisely for this reason one needs to disentangle $\delta m$ from the $1 /\left(a N_{S}\right)$ correction carefully, in order to isolate the $d=1$ renormalon.

The $\log$-terms in $\Delta_{n}^{(1,2)}$ are due to the running of $\alpha$ according to the $\beta$-function. In the lattice scheme the first three $\beta$-function coefficients $\beta_{0}, \beta_{1}$ and $\beta_{2}$ have been calculated [22]. The lack of knowledge of higher $\beta_{j}$ is leviated by the fact that these do not multiply the leading $f_{n}, f_{n-1}, f_{n-2}, f_{n-3}$ coefficients: at sufficiently high $n$, the $f_{n}$ grow factorially and therefore subleading terms become strongly suppressed.

Eq. (B.3) is used for a global fit to all orders and all data from all our lattice volumes (see Table I of [ㅁ] ). To illustrate the adequacy of the ansatz, in fig. W we compare the fit to the unsmeared triplet data, for the case of $n=9$. Note that the ansatz requires only four parameters per order, yet it results in reasonable values $\chi^{2} / N_{\mathrm{DF}} \approx 1.29$ and 1.46 for smeared and unsmeared triplet data, respectively.

Our main finding is shown in fig. [1, where we confront the infinite volume extrapolated data for $c_{n} /\left(n c_{n-1}\right)$ with the theoretical prediction, eq. (‥6). Since smearing is a local operation that has little effect on long-range physics, the smeared and unsmeared data should exhibit the same large $n$ behaviour, that is dictated by the infrared renormalon. This universality is supported by our data, as fig. $D$ illustrates. The octet representation data are also fully compatible with these findings, as we will detail in [प4]]. 


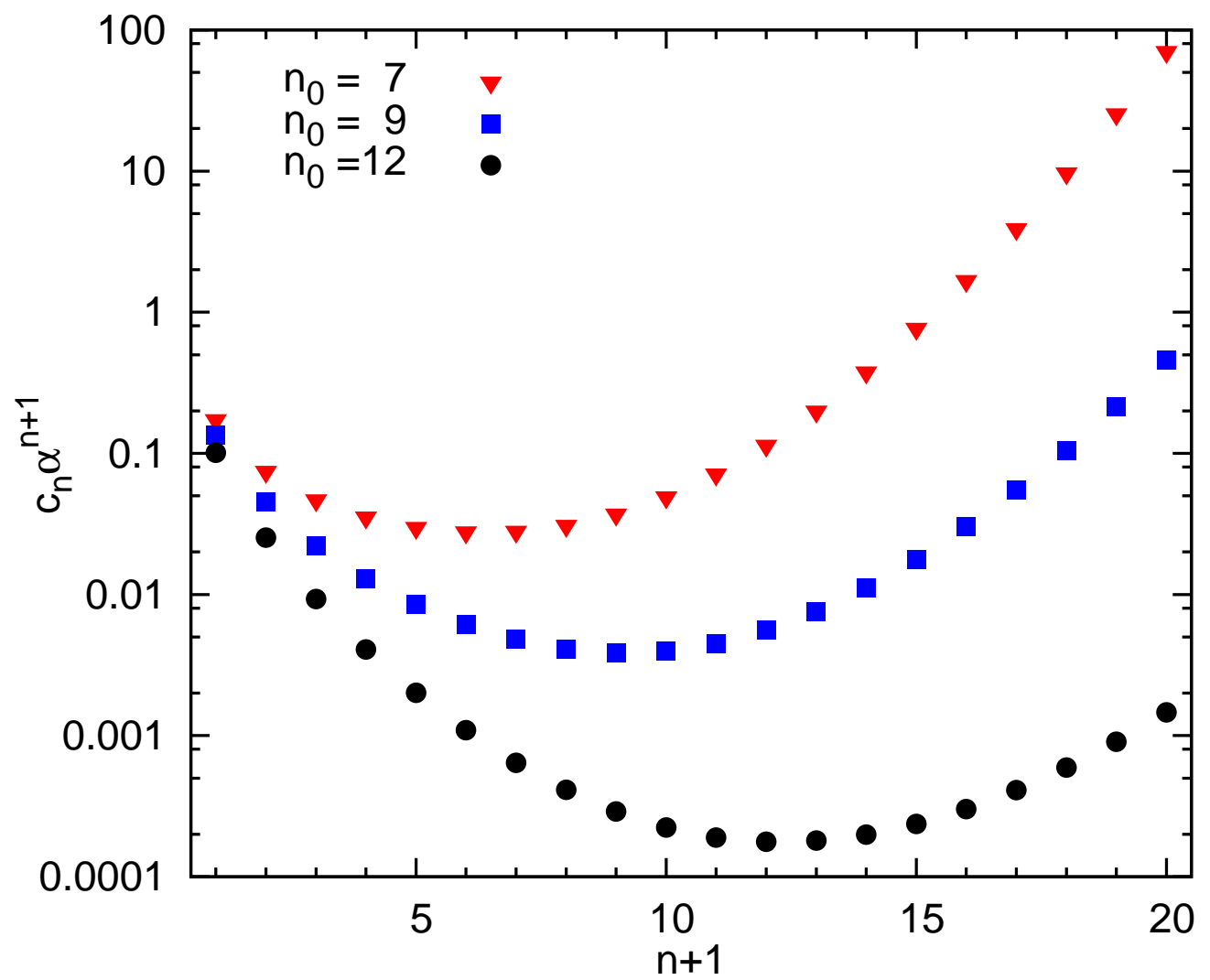

Figure 3: Contributions to the sum eq. (2.4) for three different values of $\alpha=1 /\left(\left|a_{d}\right| n_{0}\right)$, chosen such that $n_{0}=7,9$ and 12 .

In order to visualize the asymptotic behaviour of the perturbative expansion, in fig. [3 we display the order-by-order contributions to the sum eq. (2.4). For $n_{0}=7,9$ and 12, three different values of $\alpha=1 /\left(\left|a_{d}\right| n_{0}\right)$ are chosen (note that $n_{0}=7$, corresponding to $\beta=3 /(2 \pi \alpha) \approx 5.85$ or $a^{-1} \approx 1.6 \mathrm{GeV}$, is within the range covered by non-perturbative lattice simulations). The contributions indeed become minimal at orders close to the predicted values $n_{0} \approx n+1$.

With the infinite volume coefficients $c_{n}$ at hand, we can compute the normalization of the pole mass renormalon, see eq. ([2.5). For smeared triplet data we get $N_{m}^{\text {lat }}=18.6(4)$, compared to $N_{m}^{\text {lat }}=19.0(3)$ for the unsmeared case. As $\Lambda_{\overline{\mathrm{MS}}} N_{m}^{\overline{\mathrm{MS}}}=\Lambda_{\text {lat }} N_{m}^{\text {lat }}$, this translates to 0.65(2) in the $\overline{\mathrm{MS}}$ scheme. This agrees remarkably well with the estimate, $N_{m}^{\overline{\mathrm{MS}}} \approx 0.62$ [23], 24], from an expansion in the $\overline{\mathrm{MS}}$ scheme up to $\mathscr{O}\left(\alpha^{3}\right)$. Hence, the $d=1$ renormalon manifests itself in a lower bound of $\sim 0.65 \Lambda_{\overline{\mathrm{MS}}}$ for the precision with which one can determine the heavy quark pole mass.

\section{Summary}

Within the framework of NSPT, we have computed the static self-energy of SU(3) gauge theory in four spacetime dimensions to $\mathscr{O}\left(\alpha^{20}\right)$ in the lattice scheme. Simulations on various lattice volumes and a careful treatment of finite size effects are vital for our main finding, the onset of a factorial growth of the self-energy coefficients at an order $n \approx 9$, in agreement with the theoretical renormalon prediction. 


\section{Acknowledgments}

We benefited from discussions with V. Braun, F. Di Renzo, M. García Pérez, H. Perlt, A. Schiller and C. Torrero. The simulations were carried out on Regensburg's Athene HPC cluster and at the Leibniz Supercomputing Centre in Munich. C.B. acknowledges support by the Studienstiftung des deutschen Volkes and by the Daimler und Benz Stiftung. This work was supported by DAAD (Acciones Integradas Hispano-Alemanas D/07/13355), DFG SFB/TR 55, the EU ITN STRONGnet grant 238353, the Spanish grant FPA2010-16963, and the Catalan grant SGR2009-00894.

\section{References}

[1] F. J. Dyson, Phys. Rev. 85 (1952) 631.

[2] G. 't Hooft, in Proc. Int. School: The whys of subnuclear physics, Erice 1977, ed. A. Zichichi (Plenum, New York, 1978).

[3] M. Beneke, Phys. Rept. 317 (1999) 1 [arXiv:hep-ph/9807443].

[4] F. David, Nucl. Phys. B 209 (1982) 433.

[5] I. M. Suslov, Zh. Eksp. Teor. Fiz. 127 (2005) 1350 [arXiv:hep-ph/0510142].

[6] V. Zakharov, Nucl. Phys. Proc. Suppl. 207 (2010) 306 [arXiv:1010.4482 hep-ph].

[7] F. Di Renzo, G. Marchesini, P. Marenzoni and E. Onofri, Nucl. Phys. B Proc. Suppl. 34 (1994) 795.

[8] F. Di Renzo, E. Onofri, G. Marchesini and P. Marenzoni, Nucl. Phys. B 426 (1994) 675 arXiv:hep-lat/9405019].

[9] F. Di Renzo and L. Scorzato, JHEP 0410 (2004) 073 [arXiv: hep-1at/0410010.

[10] R. Horsley, G. Hotzel, E. -M. Ilgenfritz, Y. Nakamura, H. Perlt, P. E. L. Rakow, G. Schierholz and A. Schiller, PoS LATTICE2010 (2010) 264 [arXiv:1010.4674 hep-lat]].

[11] C. Bauer, G. S. Bali and A. Pineda, [arXiv:1111.3946 [hep-ph]].

[12] M. Beneke, Phys. Lett. B 344 (1995) 341 [arXiv:hep-ph/9408380].

[13] C. Torrero and G. S. Bali, PoS LATTICE2008 (2008) 215 [arXiv:0812.1680 hep-lat]].

[14] G. S. Bali, C. Bauer, A. Pineda and C. Torrero, in preparation.

[15] G. 't Hooft, Nucl. Phys. B 153 (1979) 141 .

[16] G. Parisi, in Proceedings of Progress in Gauge Field Theory, Cargese 1983, ed. G. 't Hooft et al., 531 (Plenum Press, New York, 1984).

[17] M. Lüscher and P. Weisz, Nucl. Phys. B 266 (1986) 309.

[18] A. González-Arroyo and C. P. Korthals Altes, Nucl. Phys. B 311 (1988) 433.

[19] C. Bauer and G. Bali, PoS LATTICE2010 (2010) 221 [arXiv:1011.1165 hep-lat].

[20] C. Morningstar and M. J. Peardon, Phys. Rev. D 69 (2004) 054501 [arXiv:hep-1at/0311018].

[21] H. D. Trottier, N. H. Shakespeare, G. P. Lepage and P. B. Mackenzie, Phys. Rev. D 65 (2002) 094502 arXiv:hep-lat/0111028.

[22] A. Bode and H. Panagopoulos, Nucl. Phys. B 625 (2002) 198 [arXiv:hep-1at/011021].

[23] A. Pineda, J. Phys. G 29 (2003) 371 [arXiv:hep-ph/0208031].

[24] T. Lee, Phys. Rev. D 67 (2003) 014020 [arXiv:hep-ph/0210032]. 